\title{
¿Cambios en la racionalidad de productores tradicionales?: Estrategias de producción vitivinícolas en Chilecito
}

\section{Changes in the rationality of traditional producers?: Production strategies of vine in Chilecito}

\author{
Alejandro R Carrizo y Alejandro G. Manzo \\ UNdeC, Departamento de Ciencias de la Educación y la Salud, F5360CKB Chilecito, Argentina
}

\begin{abstract}
Resumen. la inserción de Argentina al sistema mundial capitalista no se realizó en los siglos XIX y XX a partir de un proceso plenamente lineal y homogéneo. El territorio nacional presenta así un mosaico de realidades agrarias con prácticas y racionalidades diferenciadas. La eco-región Catamarca-La Rioja se ha estructurado históricamente a través de una configuración social en la que prevalecen los microfundios y una economía tradicional orientada a la satisfacción de necesidades familiares o del mercado local. En este contexto, el sector vitivinícola posee un lugar destacado y con buena proyección a futuro si se advierte que, en los últimos años, sus agentes productivos más dinámicos han incorporado nuevas tecnologías y técnicas de producción ¿Cómo estos cambios han impactado sobre el universo de pequeños productores tradicionales? Esta ponencia, siguiendo a Bourdieu, procura aportar elementos que ayuden a dilucidar este interrogante a partir del análisis de las estrategias de producción de pequeños productores vitivinícolas chileciteños. Este análisis se realiza tomando como marco de referencia empírico 67 entrevistas realizadas en Anguinán, Malligasta y Tilimuqui entre 2010 y 2013. Los resultados observados posibilitan advertir de la existencia de un grupo de productores tradicionales con una incipiente racionalidad empresarial que amerita una intervención diferenciada.
\end{abstract}

\begin{abstract}
The insertion of Argentina into the capitalist world system was not performed in the nineteenth and twentieth centuries from a fully linear homogeneous process. The country thus has a mosaic of agricultural practices and differentiated realities rationalities. The eco-Catamarca-La Rioja region has historically been structured through a social configuration in which prevail microfundios and a traditional economy oriented to the satisfaction of the family or the local market needs. In this context, the wine sector has a prominent place with good projection in the future if it is noted that, in recent years, most dynamic productive agents eco-region have incorporated new technologies and production techniques How these changes have impact on the world of small traditional producers? This paper, from the theoretical categories of Bourdieu, seeks to provide elements that help to elucidate this question by analyzing the "production strategies" chileciteños small wine producers. This analysis is performed using as a frame of reference empirical 67 interviews in Anguinán, Malligasta and Tilimuqui between 2010 and 2013 as part of a research project of the UNdeC. The observed results warn of the possible existence of a group of traditional producers with a fledgling business rationale that merits a differentiated intervention.
\end{abstract}

\section{Introducción}

Vista desde lejos, la fotografía del campo argentino proyecta la imagen de una producción agraria de punta que le permite insertarse en los principales mercados del mundo. Moldeada con los últimos avances de la denominada "segunda revolución verde", esta producción se reproduce a partir de un nuevo paquete tecnológico ordenado en torno al cultivo intensivo de semillas transgénicas, agroquímicos y maquinarias de siembra directa [1].

Esta imagen se reproduce también sobre el entramado de agentes productivos que le da vida. En efecto, en esta escala, el sector primario aparece configurado por un conjunto de agentes que, liderados por los pooles de siembra y grandes terratenientes, producen toneladas de granos para la exportación y se adaptan de manera relativamente veloz a los cambios que la economía global les impone para poder competir de forma eficiente [2].

Ahora bien, vista más de cerca la realidad agraria nacional luce distinta mostrando una gran heterogeneidad estructural. En este se pronuncian Teubal y Palmisano al observar que el "campo" argentino:

"abarca un espectro muy grande de explotaciones, que se extiende desde medianos productores cuya organización responde a la lógica empresarial en la zona pampeana hasta los tradicionales chacareros de la misma zona, así como también productores familiares y empresariales de regiones extra pampeanas [3]"1.

\footnotetext{
${ }^{1}$ En el otro extremo del espectro "están el campesinado y las comunidades indígenas, así como también una parte importante
} 
Uno de los lugares donde puede percibirse con mayor intensidad estos contrastes es en la ecoregión CatamarcaLa Rioja. De clima árido y semiárido, fue excluida del proceso de modernización que supuso el modelo agroexportador a partir del cual el país se incorporó de manera dependiente al sistema económico mundial y aún hoy, 150 años después, conserva reductos agropecuarios que se reproducen a partir de lógicas ajenas al capitalismo [4].

Esta ponencia procura precisamente reflexionar sobre estas lógicas en el sector vitivinícola; más específicamente en aquel anclado en el valle chileciteño. La elección de este valle no es inocente y obedece a su particular estructuración socio-económica. Efectivamente, en éste coexisten productores empresariales y tradicionales agrupados mayormente en zonas geográficas distintas según sus posibilidades de acceso o no a aguas subterráneas y técnicas de riego presurizado [5].

Más específicamente aún, se focaliza en las localidades de Malligasta, Anguinán y Tilimuqui en las que se concentran productores del segundo tipo ¿Cuáles son sus principales estrategias productivas y qué las diferencian de aquellas propias de los productores empresariales? ¿Constituyen un todo homogéneo o es posible, a su vez, percibir hacia su interior sub-grupos con racionalidades diferenciadas?

A fin de responder estos interrogantes, el trabajo se divide en tres grandes partes. En primer lugar, introduce los elementos teóricos-metodológicos que le dan sustento presentando una tipología de modelos de producción agropecuaria (vgr. familiar vs empresarial) en base a los desarrollos de Saal, Barrientos y Ferrer (2004); en segundo lugar, muestra empíricamente las características socio-productivas de los agentes vitivinícolas de las localidades señaladas; en tercer lugar, analiza las estrategias productivas de aquellos que poseen una superficie mayor o menor a 5 hectáreas con el objeto de advertir de la posible existencia de un sub-grupo de pequeños productores chileciteños cuyas prácticas comienzan a alejarse de las tradicionales.

\section{Estrategias productivas: Modelos empresarial vs familiar/tradicional}

Desde un enfoque estructuralista, se parte de considerar al capitalismo como un modo de producción que ha dado lugar a un sistema mundial en el que se integran de manera diferenciada distintas economías de producción asimétricas entre sí [6].

En este proceso de integración, advierte Poulantzas, las clases, fracciones de clase y las lógicas a partir de las cuales éstas se estructuran en una etapa social determinada no desaparecen de una vez y para siempre en una etapa posterior sino que subsisten, se redefinen y empiezan a reproducirse de conformidad a las relaciones de producción que ésta última supone. Dicho de otra manera, en una formación social concreta -a diferencia

de la agricultura familiar, (...) que serían los más afectados por el embate del neoliberalismo, el avance sojero y las políticas estatales que promovieron la expansión de esta oleaginosa en las últimas décadas (Teubal y Palmisano, 2011:199)". de lo que acontece en un modelo de producción purocoexisten sectores con distintos niveles de desarrollo e integración a la división internacional del trabajo hegemónica en un dado período histórico [7].

Visto así y en concordancia con una prolífera línea de investigación latinoamericana, este artículo presupone que muchos de los desequilibrios existentes en los mercados de la región están asociados a una heterogeneidad sectorial marcada, propia de regímenes de acumulación de países periféricos y dependientes como los del continente [8].

Desde sus orígenes, el enfoque estructuralista distinguió en Argentina un sector moderno e integrado a la economía global y otro tradicional anclado a la reproducción de las economías locales o regionales. A partir de la década de los 80 y, especialmente, en la de los 90 -cuando la globalización neoliberal modificó velozmente la conformación del mercado nacionaldistintos autores advirtieron de la compleja interrelación existente entre estos sectores. Esta ponencia toma como punto de partida ésa distinción aunque advirtiendo desde el inicio los límites de considerarlos como compartimentos cerrados y perfectamente definidos [9]. Precisamente, y como ya se señaló, ésta busca exhibir posibles fragmentaciones hacia el interior de los productores vitivinícolas tradicionales.

De igual modo, estos autores observaron que las primeras versiones de este enfoque, centradas en una perspectiva materialista, tendieron a restar trascendencia al carácter constructivo de la conducta humana. Dicho en otros términos, estas versiones tomaron como criterio de diferenciación de estos sectores las condiciones objetivas a partir de las cuales éstos reproducían su vida material; esto es, para definirlos se focalizaron en las distintas posiciones que éstos ocupaban en un modo de producción determinado [7].

Desde una postura diferente y a los fines de efectuar dicha diferenciación, se resaltaron aspectos culturales. Así, la principal distinción entre los sectores moderno y tradicional se ubicaría en su particular cosmovisión del mundo. Weber, por ejemplo, observó que en el centro mismo del capitalismo y consecuentemente del primer sector, se encuentra un tipo de racionalidad que se ordena en torno a la noción de eficiencia; es decir, de la elección de los medios más adecuados para obtener el máximo beneficio al menor costo posible. El concepto central en este tipo de ethos empresarial es, dice Habermas, la decisión entre cursos alternativos de acción que el agente enderezada a fin de la realización de un propósito dado y racionalmente sopesado [10].

Bourdieu, a quien se sigue en esta ponencia y quien se autodefine como "estructural-constructivista", procura sintetizar ambas tradiciones a partir de los conceptos de campo y habitus [11]. El habitus, expresan Escalante, Sanchez y Carral, recoge la interacción entre la historia social y la del individuo, la historia subjetiva y la colectiva, lo cual es fundamental a la hora de comprender la racionalidad a partir de la cual los campesinos y productores familiares construyen su identidad y dan sentido al mundo que los rodea [12]. Para ellos, advierte Barbetta, la agricultura lejos de presentarse como una forma de negocio aparece como unaforma de vida. 
"Los campesinos obtienen de la tierra su identidad (...) la tierra está unida a su propietario, pues de ella dependen sus costumbres, su cultura y sus formas de trabajar; estas últimas se instituyen en base a una relación que establece a la tierra y al campesino como cosas idénticas. Expresiones sostenidas por los campesinos y sus organizaciones como la tierra es la vida para el campesino o no hay tierra sin hombre, la tierra hace al hombre, imprimen un sello particular al derecho a la tierra [13]".

Es importante aclarar que para esta matriz teórica tanto las prácticas de los productores empresariales como aquellas de los tradicionales son fruto de una racionalidad "estratégica". Develar el carácter estratégico de las prácticas sociales no importa suponer que éstas son el resultado de una intencionalidad totalmente deliberada sino del sentido "práctico"2; ese sentido de juego particular y contextualizado que se adquiere en la actividad social misma. Las prácticas, observa Bourdieu, son estratégicas "lo que quiere decir que son finales en el sentido de que son finalizadas; pero su finalidad es normalmente objetiva, sin intención expresa, una paradoja que tiene su origen en la racionalidad implícita del habitus que (...) tiene razones que la razón ignora. Hablar en términos de estrategias quiere decir que las prácticas no son el resultado de un plan reflexionado sino el resultado de una maestría o una lógica práctica [14]”.

Interesa aquí analizar un tipo concreto de estrategias: las "estrategias productivas" a partir de las cuáles los productores chileciteños ordenan su producción vitivinícola. Más técnicamente y siguiendo a Saal, Barrientos y Ferrer, éstas "son los modos en que se utilizan o instrumentan los recursos productivos y las relaciones con los mercados [15]". Se parte de presuponer que este análisis permitirá reflexionar sobre sus características socio-productivas y compararlas, a partir de los modelostipos que a continuación se presentan, con aquellas propias de los productores modernos o empresariales.

A los fines de cumplimentar con este análisis la presente ponencia utiliza un corpus de datos recolectado en el marco de un proyecto de investigación de la UNdeC -PFIP COFECYT- ${ }^{3}$. Este corpus se confeccionó entre los años 2010 y 2013 principalmente a partir de 67 entrevistas realizadas en Anguinán, Malligasta y Tilimuqui a pequeños productores vitivinícolas. Este se completó con información recabada a través de talleres y mesas de diálogo entre técnicos del proyecto y productores de las tres localidades bajo estudio, entrevistas a representantes claves de organismos públicos vinculados a la producción vitivinícola, observación de las plantaciones y otras fuentes estadísticas secundarias (vgr. provenientes del INTA, CONSUAGUA, Dirección Provincial de Estadísticas La

\footnotetext{
${ }^{2}$ Somos sujetos prácticos, recuerda Bourdieu (1998:91), "en las tres cuartas partes de nuestras acciones".

3 "Manejo eficiente del riego en el cultivo de vid y su impacto en la producción de minifundistas del valle Antinaco - Los Colorados", Informe de la Actividad 8 Economía: "Desarrollo de Estrategias para una Adecuada Gestión y Uso Eficiente del Agua".
}

Rioja, etc.). Los datos recolectados, como se observa en los títulos siguientes, se analizan a partir de técnicas metodológicas cuantitativas y cualitativas.

\section{Caracterización socio-demográfica de Malligasta, Anguinán y Tilimuqui}

Chilecito conforma una de las dieciocho subdivisiones políticas de la provincia de La Rioja (Argentina), se encuentra ubicado al centro oeste provincial y compone la región del Valle del Famatina. El valle tiene una extensión de $25 \mathrm{~km}$, con montañas que alcanzan entre 2000 y 6.250 metros de altura. Este valle se extiende entre las sierras del Famatina al oeste y la sierra del Velazco al este. En los nevados del Famatina nacen algunas importantes corrientes, como los ríos Famatina, Durazno y Miranda. Aunque hidrográficamente es un sistema endorreico, las posibilidades de utilización de las aguas superficiales y las potentes napas subterráneas, han permitido el desarrollo de una agricultura promisoria [16].

Chilecito tiene una superficie de $4.846 \mathrm{~km}^{2}$ y una densidad poblacional de $10,2 \mathrm{hab} / \mathrm{km}^{2}$ (año 2010). Se estima que para 2014 viven en el departamento aproximadamente 54.390 habitantes, de los cuales 13,2\% lo hacen en zonas rurales. Según datos oficiales, un $29,85 \%$ de la población es económicamente activa. La distribución de la población ocupada se determina por tres grandes sectores: un cuarto de ella trabaja en la Administración Pública y aproximadamente un $14 \%$ en el sector comercial e igual participación para el agrícola [17].

La producción del valle es principalmente agrícola, donde los cultivos de vid, olivo y nogal son los de mayor importancia en ese orden. A la producción primaria se asocia la industria de vinos y aceites y otras menores de tipo vitivinifrutícola.

En 1970 el tamaño medio de las explotaciones agrícolas era de 1,6 hectáreas, en la provincia de La Rioja; el $93 \%$ de los predios tenía menos de 4 ha y el $52 \%$ menos de media hectárea. En esa misma década se trató de incorporar a los minifundistas riojanos a los beneficios de la colonización entregándoles parcelas de 9 ha con toda la infraestructura y equipamiento necesarios para producir. Sin embargo los cambios de gobierno de esa década, los vaivenes económicos del país y los criterios políticos-sociales considerados para distribuir las tierras, ahogaron las posibilidades de crecimiento sostenido del sector agrícola [18].

Otros hitos que marcarán el crecimiento del sector agrícola llegarán en los albores de la década los ochenta a finales del siglo pasado. Las leyes de promoción industrial y de desarrollo económico (que preveía utilizar recursos impositivos provenientes de grandes sectores económicos nacionales), generaron un marco jurídico orientado a potenciar la industria y el agro en las provincias de $\mathrm{La}$ Rioja, San Juan, San Luis y Catamarca.

Se puede remarcar que la puesta en marcha de estas políticas, como ejes del nuevo proceso político, provocaron profundos cambios en el sector agrícola provincial en el que se empezaba a configurar un nuevo modelo productivo basado en una agricultura intensiva en trabajo y capital, con nuevas modalidades de riego y novedosos modos de 
Tabla 1. Modelos de producción empresarial y familiar o tradicional.

\begin{tabular}{|c|c|c|}
\hline Dimensiones/modelos & Modelo empresarial & $\begin{array}{l}\text { Modelo } \\
\text { familiar/tradicional }\end{array}$ \\
\hline Objetivos & Maximizar beneficios & $\begin{array}{l}\text { Maximizar ingresos y } \\
\text { estabilidad familiar. El } \\
\text { trabajo no forma parte } \\
\text { de los costos de } \\
\text { producción. Énfasis en } \\
\text { la continuidad productiva }\end{array}$ \\
\hline $\begin{array}{l}\text { Disponibilidad de } \\
\text { recursos }\end{array}$ & Adecuada & Insuficiente \\
\hline $\begin{array}{l}\text { Vinculación con los } \\
\text { mercados }\end{array}$ & Satisfactoria & $\begin{array}{l}\text { Insatisfactoria. Bajo } \\
\text { poder de negociación } \\
\text { individual }\end{array}$ \\
\hline $\begin{array}{l}\text { Organización del } \\
\text { trabajo }\end{array}$ & $\begin{array}{l}\text { Predominio del } \\
\text { trabajo } \\
\text { asalariado }\end{array}$ & $\begin{array}{l}\text { Predominio trabajo } \\
\text { familiar.Trabajo } \\
\text { asalariado solo } \\
\text { complementario }\end{array}$ \\
\hline Dirección del Proceso & $\begin{array}{l}\text { Organización } \\
\text { centralizada. } \\
\text { Dirección y trabajo a } \\
\text { cargo } \\
\text { de distintas personas }\end{array}$ & $\begin{array}{l}\text { Dirección y trabajo a } \\
\text { cargo } \\
\text { de la familia }\end{array}$ \\
\hline $\begin{array}{l}\text { Organización de la } \\
\text { Producción }\end{array}$ & $\begin{array}{l}\text { Tendencia a la } \\
\text { especialización y las } \\
\text { prácticas fácilmente } \\
\text { tandarizadas, a fin } \\
\text { de eliminar las } \\
\text { decisiones "en el } \\
\text { terreno" o "sobre la } \\
\text { marcha" }\end{array}$ & $\begin{array}{l}\text { Tendencia a la } \\
\text { diversificación, } \\
\text { capacidad de } \\
\text { decisiones inmediatas } \\
\text { adecuadas al alto } \\
\text { grado de } \\
\text { imprevisibilidad del } \\
\text { proceso productivo. }\end{array}$ \\
\hline $\begin{array}{l}\text { Tecnologías } \\
\text { seleccionadas }\end{array}$ & $\begin{array}{l}\text { Orientadas a } \\
\text { incrementar la } \\
\text { productividad del } \\
\text { trabajo y a reducir la } \\
\text { mano de obra } \\
\text { permanente }\end{array}$ & $\begin{array}{l}\text { Definidas de acuerdo a } \\
\text { la disponibilidad de } \\
\text { mano de obra familiar }\end{array}$ \\
\hline Origin de los insumos & $\begin{array}{l}\text { Total dependencia de } \\
\text { los insumos } \\
\text { comprados, de origen } \\
\text { crecientemente } \\
\text { industrial }\end{array}$ & $\begin{array}{l}\text { Tendencia a un mayor } \\
\text { empleo de insumos } \\
\text { producidos en } \\
\text { unidad }\end{array}$ \\
\hline
\end{tabular}

cultivos y labranza relacionados entre otras cosas, con la capacidad de los agricultores de incorporar tecnologías, con la capacidad de inversión y con el tamaño de las unidades productivas [19].

A pesar de la presencia de nuevos actores en el sector, los productores tradicionales, que se encontraban en el valle no vieron afectadas sus lógicas de trabajo, en tanto los nuevos productores se ubicaron en zonas donde gracias a la tecnología, pudieron irrigar sus campos utilizando agua subterránea y sistemas de riego presurizados.

Los productores tradicionales se ubicaron principalmente en pequeños poblados satélites alrededor de la ciudad de Chilecito, posiblemente porque el diseño de los canales de riego contribuyó en la configuración del mapa productivo de la zona. Los otros oasis, distantes de la ciudad también se ubicaron en proximidades a los causes de los ríos y por donde la infraestructura de canales les proveyó del vital elemento. Mientras tanto, los productores que llegaron al valle alentados por las leyes de desarrollo económico y de promoción industrial, utilizaron tierras ubicadas hacia al sur, siguiendo un viejo diseño de colonización agrícola, independizados de la necesidad de radicarse próximo a los escasos cursos de agua.

Las localidades bajo estudio de Malligasta, Anguinán y Tilimuqui, están distantes de la ciudad de Chilecito un máximo de 5 kilómetros. Actualmente están prácticamente integradas a la ciudad, en tanto reciben los servicios públicos usuales de las zonas urbanas (infraestructura, salud, educación, comunicaciones y energía) y en los últimos años se observa el avance de la ciudad por encima de la zona rural, la cual va transformando su faz productiva por una residencial, donde conviven pequeños aglomerados urbanos rodeados de fincas de vides, olivos y frutales. 


\section{Caracterización socio-productiva de los productores vitivinícolas}

\subsection{Tierra y riego}

Uno de los factores productivos por excelencia. En el valle la superficie media es de 6,8 hectáreas (año 2013) lo cual aplica importantes limitaciones tanto a nivel productivo como en los modos de cultivo implementados por los productores.

Lo más destacable es que hasta un $70 \%$ de los productores relevados tiene terrenos con menos de 5 hectáreas. De ellos, más de la mitad tienen terrenos de hasta una hectárea.

Los regímenes de tenencia de tierra no evidencian situaciones problemáticas, en tanto desde el gobierno se iniciaron programas de saneamiento de títulos, generando que apenas más de un $10 \%$ de los pequeños productores se encuentren en procesos de regularización de sus derechos sobre la tierra.

Vinculado a la explotación de este factor, es característico que un $70 \%$ de los productores con menos d 5 ha tenga derecho a riego en su terreno. Estos derechos refieren a la facultad de utilizar agua para cultivo de sus tierras. El agua es provista por canales y el sistema de riego es a manto o superficial.

A medida que aumenta la superficie del terreno este porcentaje disminuye hasta alcanzar un $21 \%$. En general, los derechos de agua, expresados en horas de riego, son insuficientes para abastecer la totalidad de la superficie disponible.

Aquellos productores con pequeños terrenos, suelen producir combinando agua de riego con la provista por redes de agua potable. También es habitual que los esos productores compren agua de riego disponible de otros fundos que no la utilizan.

Del relevamiento surge que un $93 \%$ de los encuestados, manifiestan que riegan a manto y solo un $4 \%$ implementó riego tecnificado.

\subsection{Labores culturales: Mano de obra y herramientas}

Entre las labores culturales habituales en los predios se pueden mencionar: arado, desmalezado, fertilización, poda y atada. Gran parte de los productores aprendieron a labrar la tierra por herencia de sus mayores.

Al menos un $28 \%$ de los productores usa tractores o animales para realizar estas labores. Un $18 \%$ de ellos tienen más de 10 herramientas menores (palas, azada, azadón, trinche, hacha, etc.).

La mano de obra es principalmente familiar. Solo un $10 \%$ de los productores tiene empleados. La época de cosecha eleva esos porcentajes hasta un $38 \%$, pero se trata de mano de obra temporal.

De todos modos, más de la mitad de los productores cuenta con mano de obra familiar para trabajar la tierra (53\%). Las principales actividades en la que se ocupa la mano de obra son riego, desmalezado y poda.

La participación de la mujer en estas actividades es apenas del $10 \%$ y en épocas de cosecha asciende a $25 \%$.

\subsection{Relación con los mercados}

Los pequeños productores de las tres localidades estudiadas deben transar operaciones en condiciones de mercados con características oligopsónicas o monopsónicas (principalmente para la agroindustria de la vid). Estas condiciones del mercado local exponen a los productores a vender sus productos a precios fijados por los compradores, quienes actuarán como precio-decisores, tratando de maximizar sus beneficios a costa de pagar precios reducidos a los vendedores.

La producción se vende un $46 \%$ como insumo industrial, un $12 \%$ se comercializa en fresco en el mercado local y en pequeñas cantidades se vende a vecinos y acopiadores. Un 33\% de los productores venden de forma mixta, es decir, proveen a la industria o a los mercados locales o a sus vecinos.

Importa destacar que aproximadamente un $21 \%$ de los productores vende su producción en fresco (en puerta de finca, en planta o mercado local), de allí es plausible suponer que para acceder a estos mercados se deben cumplir ciertos estándares de calidad y eficiencia. En este sentido el proceso de cultivo implementado se asocia con ciertas prácticas agrícolas que buscan alcanzar esos objetivos.

\subsection{Composición familiar, educación, participación social e ingresos extra-prediales}

Un $80 \%$ de los productores están casados y un $28 \%$ de ellos tiene hijos menores de 18 años. Un tercio de la población bajo estudio tiene más de 60 años y solo un $4 \%$ se compone por menores de 14 años. Un dato a destacar es que un $34 \%$ de los productores tiene su vivienda ubicada en el mismo predio donde produce.

La situación poblacional de este sector indica que la presencia de niños es escasa, además que se observa un envejecimiento progresivo.

Este comportamiento se evidencia desde la mitad del siglo XX. De todas formas, en las últimas décadas se evidencia un desplazamiento de la ciudad sobre el campo.

Este desplazamiento principalmente habitacional (se observan centros urbanos en los pueblos), ha dotado al sector rural de infraestructura y servicios públicos en la misma calidad y variedad que los prestados en la ciudad.

En más de un $90 \%$ de los encuestados se constata acceso a energía eléctrica, agua potable, TV, teléfono, alumbrado público, transporte interurbano, calles pavimentadas, escuelas públicas y asistencia primaria de salud.

Aproximadamente un $17 \%$ de los productores tiene formación académica de nivel superior y solo un $4 \%$ de los encuestados no tiene instrucción.

Es importante destacar que de la oferta educativa disponible, solo hay una escuela de nivel medio orientada a la actividad agropecuaria (en todo el departamento Chilecito existen tres escuelas Agrotécnicas, las cuales fueron creadas en los primeros años del Siglo XXI).

En el valle existen organizaciones de productores y otras instituciones estatales ocupadas de la cuestión agraria (INTA, CARPA, CONSUAGUA, etc.), pero solo un $18 \%$ de los productores participa en organizaciones de ese tipo. Un tercio de ellos recibe asesoramiento profesional 
específico y apenas un $10 \%$ forma parte de proyectos agrícolas o accede a créditos propios del sector.

Uno de los datos de mayor peso en la investigación surge a partir del relevamiento de los ingresos extraprediales.

Un $59 \%$ de los productores, también trabajan en la administración pública, un $29 \%$ lo hace en el sector privado y un $12 \%$ es beneficiario de subsidios otorgados por el Estado.

Queda evidente que la fuente de ingresos no proviene de la actividad agrícola exclusivamente. Es razonable suponer que las pequeñas parcelas de cultivo, la escasez del recurso agua y una mínima introducción de tecnología, todo ello en contexto de mercados oligopsónicos, configuren una situación que afecte la posibilidad de obtener ingresos aceptables.

Va de suyo que las condiciones físicas afectan los niveles de producción, mientras que las de mercado hacen lo suyo con los precios de los productos, obligando a estos productores a financiar sus gastos por medio de la obtención de rentas provenientes del trabajo en otros sectores.

Así, los costos de oportunidad a que se enfrentan los productores agrícolas, desincentivan esta actividad en la gran mayoría de ellos.

\section{Estrategias productivas de productores de más o menos de 5 hectáreas}

Las estrategias productivas de los productores que poseen más o menos de 5 hectáreas presentan cualidades diferenciadas. A partir de su análisis es factible configurar dos subgrupos asociados a modelos de agricultura diferentes por sus lógicas productivas, organizacionales y comerciales.

Los productores que poseen más de 5 has además de regar a manto lo hacen complementado con riego tecnificado. Poseen maquinarias y herramientas menores que facilita la realización de labores culturales con mayor eficiencia y su consecuente impacto en los costos de cultivo.

A esta disponibilidad de maquinarias y herramientas, se asocia la del recurso mano de obra, el cual no depende exclusivamente de la composición familiar, sino que puede ser contratado a terceros.

Así mismo, tanto la mayor capacitación educativa que disponen como el acceso a organizaciones ocupadas de la cuestión agrícola y a asesoramiento profesional especializado, les permiten alcanzar producciones que detentan mínimos estándares de calidad, abriendo así la posibilidad de insertarse en los mercados que no son principalmente agroindustriales.

Esto último se ve potenciado a partir de la participación en organizaciones de productores y profesionales del agro, les otorga un mayor empoderamiento y eso mejora las condiciones de negociación.

Así mismo, pertenecer a esas asociaciones genera un impacto positivo en variables vinculadas con el manejo técnico del cultivo, el aprovisionamiento de insumos y hasta el acceso a fuentes de financiamiento (estos últimos no son solamente de tipo financiero, sino también a partir de servicios de asesoría profesional).

De todo ello, es posible considerar a este grupo de productores enmarcados en una lógica empresaria, donde la gestión del proceso productivo se orienta en la eficiencia y la efectividad como estrategia para minimizar costos, pero al mismo tiempo se pretende mejorar los ingresos, con el consecuente impacto en las ganancias resultantes.

El otro grupo, de mayor tamaño, tiene como principal característica que son productores familiares, son semiasalariados y poseen pequeñas parcelas de tierra. Si bien tienen acceso al recurso agua, están descapitalizados, por lo que la mano de obra tiene una presencia primordial para llevar adelante las labores culturales.

$\mathrm{Su}$ producción no está definida de antemano y su destino es incierto, pudiendo colocarse en la agroindustria o al mercado local o para consumo propio. La organización del trabajo recae sobre el jefe de familia, quien gestiona desde la experiencia adquirida de sus antepasados.

Este grupo está aislado del acceso al crédito y del asesoramiento profesional. Su actividad agrícola tiene una fuerte connotación cultural y afectiva, estando poco dispuestos a la innovación y al cambio.

Finalmente, su capacidad de negociación es prácticame nte inexistente, los precios de sus productos serán definidos por los eventuales compradores.

Este pequeño productor está imposibilitado de elevar sus ingresos, por un lado son pocas las posibilidades de mejorar sus niveles de producción (considerando la dotación de recursos que posee) y por otro lado, el nivel de precios va a estar determinado por la calidad de su producto en tanto se atiende a las demandas del mercado.

$\mathrm{Su}$ acceso al mercado que va a depender de factores bastante imprevisibles puesto que los posibles ingresos del predio van a complementar los provenientes de su trabajo asalariado en otros sectores.

\section{Reflexiones finales}

Desde una mirada general, Argentina se caracteriza por una actividad agrícola particularmente dinámica, pero es en el interior, o en la periferia de los grandes centros productores, donde emergen lógicas y racionalidades que vinculan al hombre con la tierra de un modo distintivo.

Esta ponencia se focalizó precisamente en una de estas regiones periféricas. El análisis realizado en las localidades de Malligasta, Anguinán y Tilimuqui permite observar empíricamente las siguientes características de los productores vitivinícolas chileciteños: a) acentuado microfundismo: si bien en el valle la superficie media es de 6,8 hectáreas más de la mitad de los productores revelados tienen terrenos de menos de una hectárea; b) escaso riego tecnificado: el $93 \%$ de los encuestados manifestó que riega su producción a manto y sólo un $4 \%$ implementó técnicas de riego más sofisticadas; c) carencias marcadas de maquinarias y herramientas: un $28 \%$ de los agentes de la muestra usa en su plantación tractores o animales y únicamente un $18 \%$ de ellos tiene más de 10 herramientas menores; d) preeminencia de mano de obra familiar: sólo un $10 \%$ de los entrevistados posee empleados permanentes y un $38 \%$ los contrata para la época de cosecha; 
e) encerramiento en el mercado doméstico: los productores revelados manifiestan un bajo poder de negociación al momento de vender su cosecha que destinan a industrias, acopiadoras y vecinos del mercado local.

Bajo estas características puede afirmarse que el grueso de los entrevistados se inserta en el modelo-tipo de agricultura familiar/tradicional presentado por Saal, Barrientos y Ferrer. El estudio realizado posibilita advertir que estas condiciones objetivas de producción junto a las historias colectivas que atraviesan el valle terminan configurándose como estructurantes de modos de percibir y actuar en el mundo agrícola. Estos modos se relacionan más a una agricultura de subsistencia que de acumulación. En este sentido, Barbetta describe de modo próximo y certero las cualidades de labranza de los productores de Malligasta, Anguinán y Tilimuqui estudiados cuando dice que estos obtienen de la tierra su identidad (...) la tierra está unida a su propietario, pues de ella dependen sus costumbres, su cultura y sus formas de trabajar.

Así, cualquier programa de desarrollo que se implemente debe partir de reconocer el vínculo tierra-tiempo específico de la región que supone una pertenencia sociohistórica especial con cualidades altamente diferenciadas por encima de la tecnología y de los mercados a los que se integran.

La información recabada posibilita asimismo poner bajo un manto de duda la subsistencia de esta clase de productores en el mediano o largo plazo. Esta duda surge al menos de cuatro elementos exhibidos en la ponencia: a) envejecimiento: un tercio de la población observada posee más de 60 años; muchos de los entrevistados manifiestan que sus hijos muestran poca intención de continuar con las actividades agrícolas de la familia; b) migración: sólo un $34 \%$ de los productores tiene su vivienda ubicada en el mismo predio donde produce lo que a priori rompe en las futuras generaciones con el nexo identidad-tierra señalado; c) avance de la ciudad: más de un $90 \%$ de los encuestados tiene energía eléctrica, agua potable, $\mathrm{TV}$, teléfono, alumbrado público, transporte interurbano y calles pavimentadas lo que transforma rápidamente las prácticas y rasgos culturales antes exclusivamente rurales; d) actividades extra-prediales: casi la totalidad de los productores posee ingresos ajenos a la producción vitivinícola propiamente dicha lo que evidencia que ésta, tal cual la desarrollan actualmente, no reporta incentivos económicos suficientes para convertirse en el sostén material exclusivo de sus familias.

Ahora bien, el trabajo realizado posibilita finalmente afirmar que un pequeño porcentaje de la población analizada posee rasgos propios de un modelo-tipo de agricultura más empresarial. Este porcentaje, que se corresponde con aquellos agentes que poseen más de 5 hectáreas, demuestra un dinamismo semejante al de los productores más modernos del valle. Sus cualidades distintivas, aún no plenamente institucionalizadas, ameritan una estrategia de intervención diferenciada por parte de las autoridades.

\section{Referencias}

[1] F. Barri y J. Wahren, El modelo sojero de desa rrollo en la Argentina: tensiones y conflicos en la era del neocolinealismo de los agronegocios y el centificismo-tecnológico, 2, (2009). [En línea]. Available: http://www.ger-gemsal. org . ar/wp. . ./Barri-y-Wahren-Realidad-Eco nómica.pdf. [Último acceso: 12 2011]

[2] C. Gras, Mundo Agrario, Los empresarios de la soja: cambios y continuidades en la fisonomía y composoción interna de las empresas agropecuarias, UNLP Facultad de Humanidades y Ciencias de la Educación, 12, (2012)

[3] M. Teubal y T. Palmisano, EL conflicto agrario: características y proyecciones, Del paro agrario a las elecciones de 2009: tramas, reflexiones y debates, Atropofagia, 199, (2011)

[4] M. Rapaport, Las políticas económicas de la Argentina. Una Breve Historia, Booket, (2010)

[5] A. Carrizo, E. Robles y G. Galli, Aspectos Socio Económicos de Pequeños Productores, UNdeC, (2013)

[6] A. Giron, Argentina: su recurrente inestabilidad financiera, (2009). [En línea]. Available: http://biblioteca.clacso.edu.ar/ subida/clacso/coediciones/20100617085016/ giron.pdf .ori. [Último acceso: febrero 2013]

[7] N. Poulantzas, Poder político y clases sociales en el Estado capitalista, Siglo XXI Editores, (1998)

[8] A. Salvia, F. Stefani y G. Comas, Revista Lavboratorio, Informalidad y marginalidad en los mercados de trabajo en la Argentina de la post devaluación, 21, 20, (2007)

[9] F. Betranou y L. Casanova, Informalidad laboral en Argentina: segmentos críticos y políticas para la formalización, Oficina de País de la OIT para la Argentina, (2013)

[10] J. Habermas, Teoría de la Acción Comunicativa, Taurus, (1998)

[11] P. Bourdieu, Cosas Dichas por Pierre Bourdieu, Gedisa, (1997)

[12] G. Escalante, M. Sánchez y G. Torres, El saber tradicional y el desarrollo rural sustentable, visto desde una perspectiva cultural como premisas para una ruralidad alterna tiva, 3 (2009). [En línea]. Available: http: //www2. inecc.gob.mx/publicaciones / libros / 670 / cap6.pdf. [Último acceso: agosto 2014]

[13] P. Barbetta, Cuadernos de Antropología Social - FFyL UBA, En los bordes de lo jurídico: campesinos y justicia en Santiago del Estero, 32, 126 (2010)

[14] G. INDA, Poder, Derecho y Clases Sociales de Pierre Bourdieu, Descleé de Brouwer, 46 (2001)

[15] G. Saal, M. Barrientos y G. Ferrer, El estudio del sistema social regional: los tipos sociales agrarios. 6 (2004). [En línea]. Available: http:// agro.unc.edu.ar/ extrural/SISTEMASOCIAL.pdf. [Último acceso: marzo 2013]

[16] H. Rosa y M. Mamaní, CRICyT Catálogo de recursos humanos e informacion relacionada conla tematica ambiental en la region andina arg entina (2000) [En línea]. Available: http://www. cricyt .edu.ar / laydyot / catalogo/cdandes/ cap17.htm\#inhalt. [Último acceso: agosto 2013] 
[17] Dirección General de de Estadísticas y Sistemas de Información, Etadísticas por Departamento. Chilecito. (2014). [En línea]. Available: http:// www.larioja.gov.ar/estadistica/images/agosto2014.pdf. [Último acceso: Agosto 2014]

[18] O. Páez Oliva, La Rioja industrial, Nex Ediciones, (2005)
[19] M. Tinto y C. Liurussi, Los diferimientos impositivos: ¿una esperanza para las economías regionales? [En línea]. Available: http://www. econ.uba.ar/planfenix/novedades/Area $\% 20$ II /Los $\% 20$ difermientos $\% 20$ impositivos $\% 20-\%$ 20Tinto-Lirussi.pdf. [Último acceso: agosto 2014] 